\title{
Diagnostic Correlation of Findings of Multidetector Computed Tomography and Fine Needle Aspiration Cytology in Lung Masses
}

${ }^{1}$ Rudraksh Gupta, ${ }^{2}$ Sanyukta Gupta, ${ }^{3}$ Hemant Kumar Mishra

\begin{abstract}
Introduction: The multidetector computed tomography helps to differentiate between benign and malignant lesions.

Materials and methods: In the present study, 50 patients with the lung masses underwent computed tomography (CT) of chest followed by fine needle aspiration cytology (FNAC) these patients referred from medical and chest out patient department (OPD) and indoor patient department (IPD).
\end{abstract}

Results: A total of $36 \%$ in males and $10 \%$ in female patients belong to 61 to 80 years and 41 to 60 years respectively male to female ratio $2.8: 1$. The smoking has approximate $36 \%$ association with $35 \%$ in males and $1 \%$ females. In malignant group, $32 \%$ were squamous cell carcinoma followed by adenocarcinoma (18\%), metastases $(16 \%)$ poorly differentiated carcinomas $(12 \%)$ small cell carcinoma $(6 \%)$ and large cell carcinoma $(2 \%)$.

Conclusion: The study concludes that combination of CT and CT-guided FNAC give the final diagnosis of the lesion with extension of lesion.

Keywords: Carcinoma lung, Computed tomography, Fine needle aspiration cytology, Lung masses.

How to cite this article: Gupta R, Gupta S, Mishra HK. Diagnostic Correlation of Findings of Multidetector Computed Tomography and Fine Needle Aspiration Cytology in Lung Masses. J Mahatma Gandhi Univ Med Sci Tech 2016;1(1):15-19.

Source of support: Nil

Conflict of interest: None

\section{INTRODUCTION}

Lung tumor is the most common malignancy among males followed by GI malignancy. Smoking is the strongest of the associated factor.

Lung mass lesion includes lung carcinoma, tuberculomas with tubercular lymphadenopathy, fibroma, hamartoma, leiomyoma, aneurysm, mycetoma, infarct, lymph node enlargement and carcinoma

\footnotetext{
${ }^{1}$ Senior Resident, ${ }^{2}$ Postgraduate Student, ${ }^{3}$ Professor and Head

${ }^{1-3}$ Department of Radiodiagnosis, Mahatama Gandhi Medical College and Hospital Jaipur, Rajasthan, India

Corresponding Author: Sanyukta Gupta, Postgraduate Student, Department of Radiodiagnosis, Mahatama Gandhi Medical College and Hospital, Jaipur, Rajasthan, India, Phone: +918094603204 e-mail: sanyukta15@gmail.com
}

bronchus is by far the commonest and most important primary tumor of lung.

Computed tomography (CT) serves as a dual role in the patient suspected to have a lung carcinoma based on the plain chest radiograph. Depending on the series in the medical literature approximately $40 \%$ of all resected pulmonary nodules are malignant.

\section{MATERIALS AND METHODS}

A total of 50 patients with lung masses, who were referred to the Department of Radiodiagnosis, Mahatma Gandhi Medical College and Hospital, Jaipur, were evaluated on $\mathrm{CT}$ and biopsy was done under $\mathrm{CT}$ guidance with an aim to ascertain the role of CT in evaluation of such masses and to assess the diagnostic accuracy and safety of CT-guided fine needle aspiration cytology (FNAC).

\section{RESULTS}

\section{Demographic Profile of Lung Masses}

- Most cases with lung masses belonged to age group of 61 to 80 years $(36 \%)$ in males and 41 to 60 years $(10 \%)$ in females. An overall male-female ratio was 2.8:1 in the present study.

- Incidence of lung mass is found to be associated with smoking $(72 \%)$ with $70 \%$ being males while only $2 \%$ of study females were smoker.

\section{Lung Masses: Site and Localization}

Majority of the lesion were parenchymal (88\%), most of which were within $1 \mathrm{~cm}$ from the pleura.

\section{Lung Masses: Diagnostic Methods and Test Results}

Table 1 shows $72 \%$ malignant lesions on $\mathrm{X}$-ray while CT scan and FNAC shows $86 \%$ malignant lesions. Thus, CT scan is a better modality for screening.

\section{Cytological Diagnosis}

- In the malignant group, majority 16 (32\%) were squamous cell carcinomas, followed by adenocarcinoma $9(18 \%)$, metastases $8(16 \%)$, poorly 
Table 1: Diagnostic methods and test results

\begin{tabular}{lll}
\hline Diagnostic methods & Malignant (n\%) & Benign (n\%) \\
\hline X-ray & $36(72 \%)$ & $14(28 \%)$ \\
CT scan & $43(86 \%)$ & $7(14 \%)$ \\
FNAC & $43(86 \%)$ & $7(14 \%)$ \\
\hline
\end{tabular}

differentiated carcinomas $6(12 \%)$, small cell carcinoma $3(6 \%)$, large cell carcinoma $1(2 \%)$.

- In the benign group, the majority $3(6 \%)$ were tubercular infections.

\section{Lung Masses: Morphology}

- Size: Most lesions ranged between 4 and $8 \mathrm{~cm}$ in size.

- Contour: The 50 lesions assessed by CT were classified as smooth, lobulated or irregular, based on the contour or edge characteristics. An association was observed between irregular contour and malignancy.

- Texture.

\section{Contrast Enhancement and Densitometry}

The heterogeneity of contrast enhancement showed no statistically significant differences. But, inhomogenous contrast enhancement pattern is found to be significantly associated with malignant masses (44\%) as compared to benign lesions ( $0 \%)$.

\section{Fine Needle Aspiration Cytology}

- Computed tomography guided FNAC: Needle used was 20G. pleural pass noted in 38 cases and adequate sample was noted in 40 cases.

- Complications: Complications encountered during FNAC were pneumothorax (16\%); minor hemoptysis ( $2 \%$ ) apart from minor complications like local pain and ecchymosis.

\section{DISCUSSION}

\section{Study Group Characteristics}

Most lesions ranged between 4 and $8 \mathrm{~cm}$ in maximum size and majority (88\%) were of parenchymal origin. The size is reflection of the amount of delay between the appearance of the disease and the first symptoms and ours being a tertiary level setup. This also reflect the lack of proper screening program for early detection of lung cancer, or routine health check up using plain chest skiagram, in this region.

\section{Computed Tomography Evaluation}

\section{Morphological Characteristics}

Computed tomography, due to its increased sensitivity and better resolving power can detect subtle density changes like soft tissue, fluid, fat, air or calcification.

\begin{tabular}{lccl}
\multicolumn{4}{c}{ Table 2: Morphological characteristics } \\
\hline $\begin{array}{l}\text { Morphological } \\
\text { characteristics }\end{array}$ & $\begin{array}{c}\text { Malignant } \\
(n \%)\end{array}$ & $\begin{array}{c}\text { Benign } \\
(n \%)\end{array}$ & Total \\
\hline Solid & & & \\
\hline Homogenous & $23(95.8 \%)$ & $1(4.1 \%)$ & 24 \\
Inhomogenous & $20(76.9 \%)$ & $6(23 \%)$ & 26 \\
\hline Calcification & & & \\
\hline Central & $10(83.3 \%)$ & $2(16.7 \%)$ & 12 \\
Peripheral & $5(83.3 \%)$ & $1(16.7 \%)$ & 6 \\
Air bronchogram & $3(60 \%)$ & $2(40 \%)$ & 5 \\
\hline
\end{tabular}

- Size: Siegelman et $\mathrm{al}^{1}$ and Zeerhouni et al ${ }^{2}$ summarized that smaller lesions were more apt to be benign. Most lesions in the present study were between 4 and $8 \mathrm{~cm}$ size of the total 50 lesions in which measurements were made 43 were malignant $(86 \%)$ and 7 were benign (14\%). The reason for the observed difference was probably due to the fact that for lesion larger than $2 \mathrm{~cm}$ in diameter, the frequency of benignancy falls sharply. However size alone cannot differentiate between benign and malignant lesion.

- Contour: The 50 lesion assessed by CT were classified as smooth, lobulated or irregular, based on the contour or edge characteristics. Contour alone, has poor specificity and predictive value for malignancy, however it is quite sensitive and pulmonary masses with irregular margins should be assessed as indeterminate even though they fulfill other criteria for benignancy. ${ }^{1}$ Whereas Sones et al did not ascribe any importance to the edges, Siegelman et al, suggested that carcinomas as a group tend to have irregular edges, though edge alone is a poor predictor of malignancy as they also encountered many smooth marginated homogenous carcinomas. ${ }^{1,3}$

\section{Other Morphological Characteristics}

Characteristics studied under this heading were the degree of homogeneity of the lesions, presence and pattern of calcification, air bronchograms or air loculi. Table 2 shows the following characteristics:

Computed tomography enhancement pattern: The preponderance of inhomogenous lesions (52\%) could be explained by the fact that firstly, most of the lesions were large and secondly, a large proportion of them were malignant. Thus heterogeneity of a lesion is of little diagnostic value in itself for any prediction.

Among the homogenously enhancing lesions, 18/18 were malignant and none were benign. This finding was also observed by Yamashita et al (1995). However, they suggested that peripheral and capsular enhancement pattern, both suggested tuberculomas (or granulomas) or hamartomas. 
- Computed tomography densitometry is of little use in lesions of more than $3 \mathrm{~cm}$ in size for differentiating benign and malignant lesions.

- Visualization of gross calcification on CT scans within lung lesions does not exclude the diagnosis of bronchogenic carcinoma.

- Presence of peripheral (capsular) enhancement is strongly suggestive of granuloma or abscess.

\section{Use of Intravenous Contrast}

The maximum attenuation difference value between 20 and $60 \mathrm{HU}$ is an important indicator of malignancy. Most benign lesions have maximum attenuation difference value between 0 and $10 \mathrm{HU}$. Maximum attenuation difference values between 11 and $20 \mathrm{HU}$ should be regarded as indeterminate. Littleton et al were the first to use computerized tomography to analyze pulmonary nodules after IV contrast infusion, and suggested the use of CT to simplify the technique. ${ }^{4,5}$ The study was based on the fact that the blood supply of the malignant nodules is qualitatively and quantitatively different from that of most benign nodules, and enhancement of a nodules should depend on the amount of contrast material that enters the extravascular space of the nodules and on the degree of vascularity of the nodules as was seen on pulmonary nodules obtained after surgery or necropsy. ${ }^{6,7}$ They worked to demonstrate that enhancement of pulmonary nodules indeed can be used to differentiate benign and malignant by $\mathrm{CT}^{4}$

\section{Air Bronchograms}

Air bronchogram is an important radiologic sign of air space consolidation in which the normally invisible bronchial air column becomes visible because of contrast with surrounding tissues. ${ }^{8}$ However, air bronchograms have been considered uncommon in other types of lung cancer but suggested that when an air bronchogram or bronchiologram is detected in a small peripheral lung nodule, suspicion of malignancy should be high. ${ }^{9}$ The presence of air bronchograms or air loculi in pulmonary lesions was seen in only 5 lesions; out of which 3 proved to be benign while rest malignant. However all the lesions were of large size and the significance of this finding in small peripheral lung cancer could not be evaluated.

\section{Computed Tomography Guided FNAC}

The ideal test that distinguishes all benign lesions from malignant ones is yet to be discovered. Not all masses/ nodules can be categorized as definitely benign or malignant only by CT. In a study, could not classify 103 out of 634 nodules as definitely benign, many of which required needle biopsy for final diagnosis. ${ }^{1}$ Dahlgren and Nordenstrom were the first to use image intensified fluoroscopy to guide the skinny needle for biopsy of lung masses. Their results showed a high accuracy and a low complication rate. ${ }^{10,11}$ Haaga et al introduced CT guidance, in the year $1976 .{ }^{12}$ It soon developed into a valuable tool for sampling different areas. In lung biopsies, it has developed a much larger role than anticipated. ${ }^{13}$

\section{Advantages of Conputed Tomography}

- Versatility is one of the most valuable advantage of the CT-guided procedures. Changing of the body position of the patient may be advantageous for opening 'windows' for instruments because of organ shifting or patient comfort (changing positions creates no adverse effect on image quality). ${ }^{14}$

- Biopsy of the necrotic center of a neoplasm provides little histopathologically valuable tissue. With CT the needle can be guided to the wall of the lesion rather than to its center. ${ }^{15}$

- Computed tomography may also identify an arteriovenous malformation and thus prevent potentially hazardous biopsy. ${ }^{16}$

\section{Complications}

Studied that most common complication following percutaneous biopsy are pneumothorax and pulmonary hemorrhage. In our study, complications encountered during FNAC were pneumothorax (16\%); minor hemoptysis $(2 \%)$ apart from minor complications like local pain and echymosis. All pneumothoraxes were minor and asymptomatic. ${ }^{16}$

\section{Role of Diagnostic Modalities}

- Clinical examination, including chest X-rays, should be the primary diagnostic modality in lung masses. There should be periodic X-rays examinations, especially in high-risk group patients.

- Bronchoscopy, bronchoalveolar lavage and bronchoscopic biopsy should be reserved for cases in which involvement of major airway vocal cords and carina is suspected or in cases were CT evaluation detects 4 th to 6 th order CT-bronchus sign.

\section{ILLUSTRATIONS}

Chest skiagram PA view and NCCT chest axial image in the same patient shows partially defined opacity in Lt. upper lung field. In CT image, it was found to be consolidation with air bronchogram (Fig. 1).

Heterogeneously enhancing mass lesion in the lung (A) peripheral (B) hilar (Figs 2A and B). 

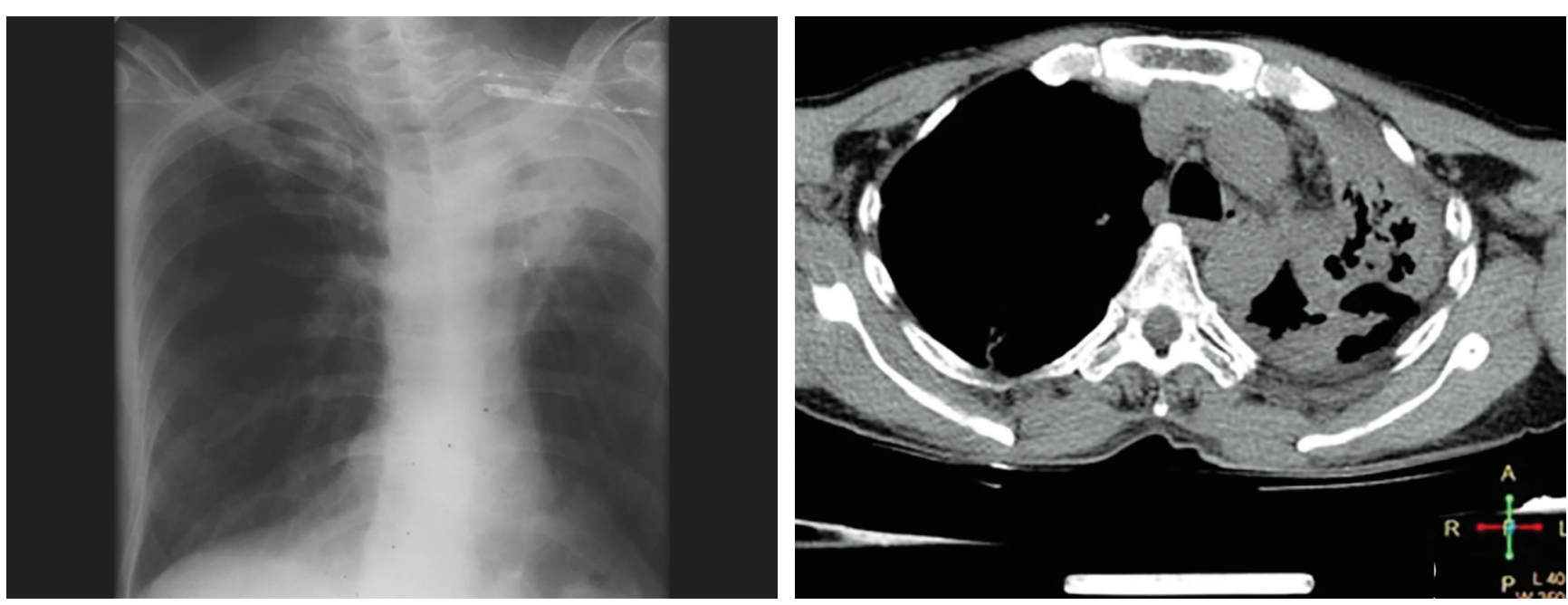

Fig. 1: On chest radiograph diagnosed as left hilar mass but on CT proved to be consolidation


Figs. 2A and B: Peripheral and hilar masses

Axial CT images in (Figs 3A and B) in these two different patients show lung masses in left upper lobe in (3A) and right upper lobe in (3B). Both of these masses show areas of central calcifications which are present. Histopathological confirmation shown squamous cell carcinoma in (3A) and poorly differentiated carcinoma in (3B) (Figs $3 A$ and B).

Axial CT images in (Figs 3 C and D) in these two different patients show lung masses in right upper lobe in (3C) and left upper lobe in (3D). Both of these masses show areas of peripheral calcifications which are present. Histopathological confirmation shown squamous cell carcinoma in (3C) and adenocarcinoma in (3D) (Figs 3C and D).

\section{CONCLUSION}

The merits of CT in intrathoracic scanning are innumerable. But the most important amongst them, which is also the area of interest of present work, is the ability to evaluate lung masses on its own and to differentiate them in terms of benign and malignant with reasonably high degree of accuracy. Moreover, it is a modality, which does not have a dead end.

\section{REFERENCES}

1. Siegelman SS, Khouri NF, LEO FP, Fishman EK. Solitary pulmonary nodule CT assessment. Radiology 1986; 160:307312.

2. Zeerhauni EA, Boukaddoum MA, Siddiky MA, et al. A standard phantom for quantative $\mathrm{CT}$ analysis of pulmonary nodule. radiology 1983;149:767-772.

3. Sones, Davis TM Jr. Hilar masses: evaluation with CT guided biopsy after negative bronchoscopic examination. radiology 1997;164:107-109.

4. Kormano M, Partanen K, Soimakallio S, Kivimäki T. Dynamic contrast enhancement of the upper abdomen: effect of contrast medium and body weight. Invest Radiol. 1983 JulAug;18(4):364-7.

5. Littleton JT1, Durizch ML, Moeller G, Herbert DE. Pulmonary masses: contrast enhancement. Radiology. 1990 Dec;177(3):861-871.

6. Milne EN, Zerhouni EA. Blood supply of pulmonary metastases. J Thorac Imaging 1987 Oct; 2(4):15-23. 



Figs $3 A$ to $D$ : Calcification patterns

7. Wright FW. The radiological diagnosis of lung and mediastinal tumor: London, Butterworth 1973.

8. Fleischner FG. Visible Bronchial Tree: A roentgen sign in pneumonic and other pulmonary consolidation. Radiology 1948;50:184-189.

9. Theros EG. Caldwell lecture: Various manifestation of peripheral pulmonary neoplasm: Am J Roentrenology 1977 June;128(6):893-914.

10. Fink I, Gamsu G, Harter LP. CT-guided aspiration biopsy of the thorax. J Computerized Assist Tomogr 1982 Oct; 6(5):958-962.

11. Nordenstrom B. Transthoracic needle biopsy. New Engl Jmed 1967;276:1081-1082.
12. Haaga JR, Alfidi RJ. Precise Biopsy Localization by CT. Radiology 1976;118:603-607.

13. Goldwasser B, Cohan RH, Dunnick NR, Andriani CC, Carsen CC 3rd, Weinerth JL. Role of linear tomography in evaluation of patients with nephrolithiasis. Urology 1989;33:253-256.

14. Herman SJ, Winton TL, Weisbrod GL, Towers MJ, Mentzer SJ. Mediastinal invasion by bronchogenic carcinoma: CT signs. Radiology. 1994 Mar;190(3):841-846.

15. Swenson D. CT guided transthoracic biopsy of mediastinal masses. J Intev Radiol 1990;5:163-164.

16. Wu M, Chern M, Liu J. Massive air leak after CT-guided lung biopsy. European Journal of Radiology 2006;60(1):15-17. 